\title{
Modelling and Performance Evaluation of Improved Access Mechanisms in a Novel Multiservice OPS Architecture*
}

\author{
Thaere Eido $^{1}$, Ferhan Pekergin ${ }^{2}$, and Tülin Atmaca ${ }^{1}$ \\ ${ }^{1}$ TELECOM \& Management SudParis, RST Dept., 9 rue Charles Fourier, \\ Evry, 91011 France \\ ${ }^{2}$ LIPN - CNRS UMR 7030, Av. J.B. Clément, Villetaneuse 93430 France \\ \{Thaere.Eido, Tulin.Atmaca\} @it-sudParis.eu, \\ Ferhan.Pekerginalipn. univ-paris13.fr
}

\begin{abstract}
Optical Packet Switching (OPS) technologies are among the most promising solutions for Next Generation Networks. In OPS networks, several mechanisms for Quality of Service (QoS) management have been developed. In this paper, we address QoS and fairness issues in a novel OPS ring architecture with a slotted synchronous transmission. The MAC protocol in the studied architecture is similar to CSMA/CA. Each node may use the available bandwidth in an opportunist manner. Therefore, the chances for each node to find available transmission resources are tightly correlated to the matrix of traffic at the other nodes. In order to remedy this problem, we elaborate two improved access mechanisms with preemptive approaches: Packet Erasing Mechanism (PEM) and Extraction and Reemission Mechanism (ERM). We evaluate the performance of the proposed mechanisms using a discrete-time analytical model. Finally, the results obtained by our model are validated and analyzed through several simulation scenarios.
\end{abstract}

Keywords: Medium Access Control (MAC), Metro Access Network Architectures, Modeling and Performance Evaluation, Quality of Service, Slotted Optical Packet Switched (OPS) Ring Networks.

\section{Introduction}

Packet ring-based networks were pioneered by the Cambridge Ring, followed by other important network architectures such as FDDI and RPR. Those networks are mainly based on electronic routers which provide satisfying performance but with relatively high cost. However, the French research project ECOFRAME is targeting a new optical Metro Access Network (MAN) [1], [2]. This network architecture aims to develop a new technological approach with lower cost compared to a pure electronic solution. It has also some added values in terms of reliability and performance. The studied network

This work was partially supported by the French ANR / ECOFRAME and by the European EuroNF research projects. 
is synchronous. Moreover, the transmission medium is subdivided into slots of equal duration. Each slot can accommodate one fixed-size optical packet.

In the scope of this paper, we address modeling and performance evaluation of optimized access mechanisms that we propose for this novel OPS architecture. We use an absolute priority scheme inside each ring node. However, it is well known that the absolute priority scheme improves the performance of the higher priority classes, but can cause excessive delays for the low-priority classes especially when the network is highly loaded [3]. Discrete time communication systems with constant slot size, including several priority management schemes, have been studied with many details in [4]. Besides, a dynamic packet transmission discipline, with multiple classes of delay-sensitive traffic and the Head of the Line (HOL) priority scheme, was proposed and analyzed in [5]. Moreover, a dynamic priority scheme with dynamic jumps has been recently investigated by analytical modeling [6]. However, while these studies have provided interesting results concerning the priority management inside a single node, none of them have taken into account the interactions through the link between several nodes at the network scale. In fact, even the absolute priority that performs a large discrimination between different classes of traffic may lack efficiency when it is applied to a ring or bus topology. Depending on the traffic matrix, some stations can have a very privileged position which allows them to monopolize a large amount of bandwidth. Hence, the priority scheme must be elaborated at the scale of the network and not only at the node scale.

Therefore, we elaborate two preemptive access mechanisms: Packet Erasing Mechanism (PEM) and Extraction and Reemission Mechanism (ERM). These mechanisms prevent the transmission of packets with high priority from being blocked by packets of lower priority sent by other ring nodes. The preemption of $\mathrm{BE}$ packets at intermediate nodes is controlled by a Queue-Length Threshold (QLT) policy. Thus, this mechanism implements a sort of dynamic priority. In the studied architecture the interaction between classes is performed through the ring. In addition to the state of local buffers, the use of the slots (link state) should also be considered in the analysis of such system. This original feature of this study extends the QLT priority scheme to the network level.

From the modeling point of view, the synchronous slotted mode that characterizes the studied architecture imposes the resort to a common service policy which is based on a cyclic admittance scheme [7]. However, in the studied ring network architecture, optical packets bypass intermediate nodes transparently. Moreover, the transmission medium is shared by several nodes. Hence, the transmission of new packets arriving to the system undergoes a possibility of blocking. After resolution of the proposed model, we provide expressions for delay and loss performance parameters. The results obtained are validated by simulation works.

This paper is organized as follows. The studied network architecture is described in section 2 . In section 3 we present the ring node function and the main ideas that inspire the proposed model. Expressions for delay measures and packet loss ratio are provided in section 4. Novel QoS mechanism (ERM) and the extension of our model for its support are presented in section 5. Section 6 gives model validation by simulation works and performance analysis of the studied network and the proposed mechanisms. Finally, section 7 concludes our work. 


\section{Studied Network Architecture}

We consider a ring network with synchronous transmission, which allows the transport of fixed-size optical packets in a slotted mode. Client traffic consists of several classes of Service $(\mathrm{CoS})$. Ring nodes communicate directly without resorting to any central station (Hub).

Each ring node (station) can receive, drop or insert packets to be transmitted on the ring. The MAC protocol in the studied network is similar to the Carrier Sense Multiple Access with Collision Avoidance (CSMA/CA) scheme [8]. The node constantly watches on the transit line (link), seeking for free slots on the ring. Network nodes read the header of all incoming packets in order to decide whether to extract or not each of these optical packets from the ring. When a node is the destination of a packet, it removes the packet completely from the ring.

In the studied architecture, the traffic coming from client networks consists of small electronic packets of variable-size. These electronic packets are collected and put together into large fixed-size optical payloads. In the following sections, we refer to this procedure as the fixed-size packet creation mechanism. Note here that we do not deal in this study with this procedure since it has been well studied in [9]. The resulted optical packets are stored in electronic queues called local buffers (one buffer for each class of service). When the current slot is free, local buffers are served according to a head of line (HOL) policy. Since we consider synchronous slotted transmission, the node can transmit an optical packet (if any) only at the beginning of a free slot.

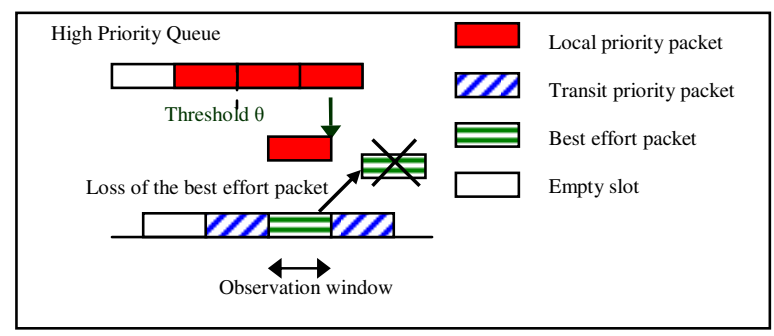

Fig. 1. Packet erasing mechanism

As for the QoS management, we propose two improved preemptive access mechanisms: the Packet Erasing Mechanism (PEM) and the Extraction and Reemission Mechanism (ERM). As shown in Figure 1, when the PEM mechanism is enabled, Best Effort (BE) packets can be dropped (preempted) at intermediate nodes in order to be replaced by packets of higher priority. We denote by $C$ the number of classes of service in the network and by $N q$ the capacity of the buffer of class $q(q=1, \ldots, C)$. Note that $q=C$ corresponds to the Best Effort class. In order to limit the preemption of BE packets, the activation of the erasing mechanism is controlled. Packets of class $q$ $(q=1, . ., C-1)$ can preempt BE packets only when the number of packets in the buffer of class $q$ is superior or equal to a threshold $\theta_{q}\left(\theta_{q}=1, . ., N_{q}\right)$. 


\section{Modeling of the OPS Ring}

\subsection{Network Model}

We consider a ring constituted by $S$ stations connected by a link. In this structure each station behaves in the same way, therefore they are characterized by the same node model but possibly with different parameters. However, the nodes in the network interacts through the link, so to describe the network behavior, the link state indicating if the slots are free or occupied, must be also determined.

Each node encloses $C$ electronic buffers in order to accommodate a number of $C$ Classes of Service $(C o S)$. CoS 1 is the class with the highest priority. We may also refer to these buffers as add buffers or local buffers. The capacity of a local buffer $q$ $(q=1, \ldots, C)$ is the number of packets $N_{q}$ which can be stored inside.

We suppose that packets of class $q$ arrive to the station following a Poisson process with a rate $\lambda_{q}(q=1, \ldots, C)$. We denote by s, the state of a slot on the link. When a slot is free, its state is 0 ; otherwise it corresponds to the $\operatorname{CoS} \operatorname{ID}(1, \ldots, C)$ of the packet transmitted in this slot.

\subsection{Computation of the State Probabilities}

Hereafter we compute the state probabilities for local buffers at the stationary state of the system. We are interested only in the state of the transmission buffers. The state of the reception buffer doesn't impact the network performance.

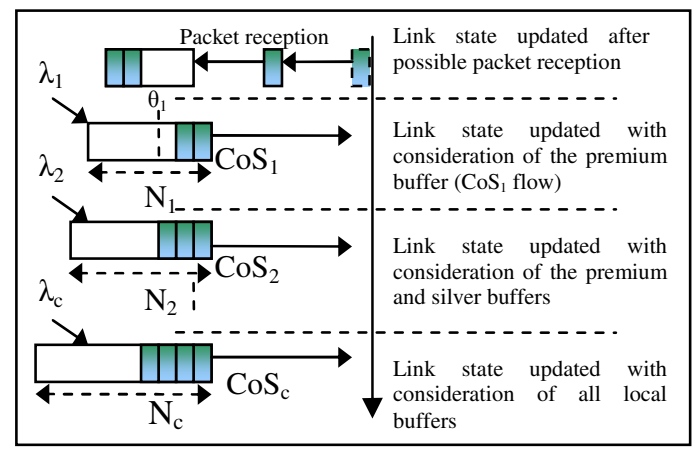

Fig. 2. Steps of slot state update (decomposition method)

The queueing policy at each node is based on HOL absolute priority. The service of clients of class $q$ depends only on the service of potential clients in the previous queues (classes 1 to $q-1$ ) and on the state of the current slot seen by the first buffer in the considered node. Hence, we decompose our system to as many parts as the number of local buffers in all ring nodes. The effect of each node is reported on the link state in a circular sequence. For a timeslot observed by a single node, the state of the link is modified by taking into account the state of the local transmission buffers, considered in a sequential way according to their priority. Moreover, at the entrance of each node, the state of the current slot is updated in order to take into account the 
traffic matrix, i.e. the probability for the potential packet carried on this slot to be destined to the current node. Figure 2 illustrates the above described decomposition.

Due to the constant slot duration, the steady state distribution of this system depends on the observation moments that we choose. For the resolution of the studied model, we observe the system state right after a possible modification of the link state, taking into account the effect of all queues in the node under consideration. So, the steady state of the system is observed at the end of each timeslot, right after the slot passes the current node and before it goes to the next node on the ring. The model obtained after decomposition using the priority is a typical discrete time queueing model with buffers of finite capacity. It can be easily shown that the associated steady state distribution exists and it is unique.

At each node, the state of the link is modified consecutively by taking into account the effect of each local buffer $q(q=0, \ldots, C)$, where $q=0$ denotes the reception buffer and $q=1, \ldots, C$ denote local transmission buffers. Let $P_{l, q}(s)$ be the probability for the current slot on the link, seen by the buffer $q$, to be in the state $s(s=0, \ldots, C)$. Hence, $P_{l, q+1}(s)$ will represent the state probability of the link after taking into account the effect of the buffer $q$.

Let $P_{q}(n)$ be the probability for the buffer of class $q$ to have $n$ packets inside at the end of the current timeslot (taking into account new arrivals and after one of its packets has been possibly moved to the server for transmission).

We denote by $\Omega_{q, n_{q}}(k)$ the conditional probability that $k(k \in\{0,1\})$ packet exits from the queue of class $q$ during the current slot when $n_{q}>0$.

$$
\left.\Omega_{q, n_{q}}(k=1)=P_{l, q}(0)+1\left(\mathrm{n}_{\mathrm{q}}\right\rangle \theta_{q}\right) \cdot P_{l, q}(C)
$$

where 1 (.) is the indicator function which takes the value 1 if the associated condition is satisfied and 0 otherwise. Clearly, the second term expresses the case where a Best Effort packet (called BE or class $C$ ) is erased to the benefit of the class $q$.

We denote by $\alpha_{q}(j)$ the probability that $j$ packets of class $q$ arrive in timeslot duration $\Delta$. When the packet arrivals is characterized by a Poisson process, $\alpha_{q}(j)=\left(\lambda_{q} \times \Delta\right)^{j} \cdot e^{-\lambda_{q} \times \Delta} / j$ !

During the computation, first, the state of the local buffer $q$ is updated taking into account the state of the link viewed by this queue. Then the state of the slot is updated taking into account the effect of $q$. The new state of the slot will be seen by the next queue (of class $q+1$ ) of the node. The last update in a node gives $P_{l, 0}(s)$ for the next node on the ring. Note here that since we are interested in the stationary state of the system, the initial values of the state probabilities (of the link and the local buffers) have no impact on the results but only on the computation time.

For the node under consideration, the state of the buffer of class $q$ changes upon the arrival of new packets or the departure (transmission) of the packet at the head of it. Therefore, this state is characterized by the following transition equations:

$$
P_{q}^{+}(n)=\left\{\begin{array}{lr}
\sum_{i+j-k=n} P_{q}(i) \cdot \alpha_{q}(j) \cdot \Omega_{q, i}(k) & \text { for } 0 \leq n<N_{q}-1 \\
\sum_{i+j \geq N_{q}} P_{q}(i) \cdot \alpha_{q}(j) \cdot \Omega_{q, i}(1)+\sum_{i+j=N_{q}-1} P_{q}(i) \cdot \alpha_{q}(j) \cdot \Omega_{q, i}(0) & \text { for } n=N_{q}-1 \\
\sum_{i+j \geq N_{q}} P_{q}(i) \cdot \alpha_{q}(j) \cdot \Omega_{q, i}(0) & \text { for } n=N_{q}
\end{array}\right.
$$


Recall that, the probabilities $P_{l, 0}(s)$ should be updated at the entry of each node depending on whether the packet at the current slot is destined or not to the current node. This update will provide the probabilities $P_{l, 1}(s)$, which characterize the link state seen by the first buffer in the node. Transition equations for this update are:

$$
\left\{\begin{array}{l}
P_{l, 1}(0)=P_{l, 0}(0)+\sum_{i=1}^{C} P_{l, 0}(i) \cdot P_{R, i} \\
P_{l, 1}(i)=P_{l, 0}(i) \cdot\left(1-P_{R, i}\right) \quad \text { for } \quad 1 \leq i \leq C
\end{array}\right.
$$

where $P_{R, i}(i=1, . ., \mathrm{C})$ is the probability for a packet of class $i$ to be destined to the considered node. For a uniform traffic in a ring with $S$ stations, $P_{R, i}=1 /(S-1),(i=1, . ., \mathrm{C})$

Finally, the state transitions for the slot, considering the buffer of class $q$, are:

Case 1: The slot is free and no packets in queue are ready for insertion.

Case 2: The queue is not empty and the slot is free.

Case 3: The slot carries a $B E$ packet, which is preempted by the PEM mechanism.

Case 4: The slot is carrying a transiting packet and no insertion event occurs.

The state transitions for the slot under the effect of a queue $q(q=1, . ., C-1)$, taking into account the state of this queue at the end of the timeslot, are described as follows:

$$
\left\{\begin{array}{l}
P_{l, q+1}(0)=P_{l, q}(0) \cdot P_{q}(0) \cdot \alpha_{q}(0) \\
P_{l, q+1}(q)=P_{l, q}(q)+P_{l, q}(0) \cdot\left(1-P_{q}(0) \cdot \alpha_{q}(0)\right)+P_{l, q}(C) \cdot\left[\sum_{i+j \geq \theta_{q}} P_{q}(i) \cdot \alpha_{q}(j)\right] \\
P_{l, q+1}(C)=P_{l, q}(C) \cdot\left[1-\sum_{i+j \geq \theta_{q}} P_{q}(i) \cdot \alpha_{q}(j)\right] \\
P_{l, q+1}(i)=P_{l, q}(i) \text { if } 0<i<C \text { and } i \neq q
\end{array}\right.
$$

Note here that a packet that arrives to the node during a slot is stored, at least till the end of the current slot, in the local buffer that corresponds to its class of service.

\section{Delay and Loss Expressions}

\subsection{Computation of Packet Loss Ratios}

In the studied architecture, packets may be lost at local buffers because of buffer overflow or, for $B E$ packets, dropped at intermediate nodes by the PEM mechanism. The Packet Loss Ratio $\rho_{q}$ is the ratio of packets which are lost because of overflow of the buffer $q$, to total generated packets of class $q$.

When there are $n$ packets in a local buffer $q$ of capacity $N_{q}$, the arrival of $k$ new packets $\left(k>\left[N_{q}-n\right]\right)$ in a timeslot of duration $\Delta$ causes the loss of $k-\left[N_{q}-n\right]$ packets. Therefore, $\rho_{q}$ can be expressed as:

$$
\rho_{q}=\sum_{n=0}^{N_{q}} \sum_{k=N_{q}-n+1}^{\infty} \alpha_{q}(k) \cdot P_{q}(n) \cdot\left[k-\left(N_{q}-n\right)\right] / \lambda_{q} \cdot \Delta
$$

Thereafter, the performance measures concerning different stations will be written with a superscript $k$. For example, the loss probability at the add buffer of class $q$ in 
the station of rank $k(k=1, . ., S)$ will be denoted as $\rho_{q}{ }^{(k)}$. This superscript will be removed whenever there will be no risk of confusion.

The total loss ratio of packets of class $q(q=1, \ldots, C-1)$ is equal to $\rho_{q}{ }^{(k)}$. As for the class $C$, packets which are dropped at intermediate nodes should also be considered.

We denote by $P_{d \mid C}{ }^{(k)}$ the conditional probability for a packet of class $C$, being transmitted on the slot, to be dropped at the intermediate station of rank $k$.

$$
P_{d \mid C}^{(k)}=1-\prod_{q=1}^{C-1} \sum_{0 \leq i+j<\theta_{q}^{(k)}}\left(P_{q}^{(k)}(i) \cdot \alpha_{q}^{(k)}(j)\right)
$$

A station of rank $v$ may send packets to the stations situated between $n \operatorname{ext}(v)=[(v+1) \bmod S]$ and $\operatorname{prev}(v)=[(v-1) \bmod S]$, where $\bmod$ denotes the modulus function. A Best Effort packet enters to the buffer of class $C$ in the station $v$ with the probability $\left(1-\rho_{C}^{(v)}\right)$. Afterwards, the probability for this packet to reach its destination (that we denote by $k$ ) can be expressed as: $\prod_{h=n e x t(v)}^{p r e v(k)}\left[1-P_{d \mid C}^{(h)}\right]$

We denote by $r_{k \mid C}^{(v)}$ the probability for a packet of class $C$ arriving to the station $v$ to be destined to the station $k$. Therefore, the total loss ratio of Best Effort packets arriving to a station $v$ can be expressed as follows:

$$
\rho_{C, P E M}^{(v)}=1-\left(1-\rho_{C}^{(v)}\right) \cdot\left[\sum_{k=(v+2) \cdot \bmod . S}^{p r e v(v)} r_{k \mid C}^{(v)} \cdot \prod_{h=\text { next }(v)}^{p r e v(k)} \cdot\left[1-P_{d \mid C}^{(h)}\right]\right]
$$

Recall that the packets sent from $v$ to $k=[(v+1) \bmod S]$ are not be affected by the erasing mechanism PEM, hence $[(v+1) \bmod S]$ is omitted in the above summation.

\subsection{Computation of the Moments of the Queueing Delay}

Each packet arriving into a local buffer should wait a time $R$ up to the beginning of the next timeslot. Afterwards, it should wait a time $W$ until it gets to the head of the queue. Because of the non-continuous availability of the link, a packet which reaches the head of the queue should wait an additional delay (called access time or $A$ ) until it finds a free slot where it is then transmitted.

The queueing delay $Q$ inside each local buffer consists of the sum of $R, W$ and $A$. Moreover, the waiting time $W$ is equal to the sum of access times of packets seen by the arriving one while entering to the system. Recall that for a queue having stationary distribution and state changes of one-step type (which is the case in our model), the distribution found at the incoming moments and left at outgoing moments are the same [10]. Hence, each packet entering (but not all arriving packets) to a local buffer sees the same distribution, as a packet which has just been retrieved from the queue.

Therefore, if we characterize the state of the system right after packet transmission moments, then the queueing delay $Q$ is expressed as: $Q=R+\left(1+X_{e}\right) \cdot A$, where $X_{e}$ is the queue length seen by a packet just before entering to the queue or just after leaving it. $R, X_{e}$ and $A$ are independent random variables, therefore:

$$
E[Q]=E[R]+\left(1+E\left[X_{e}\right]\right) \cdot E[A]
$$




$$
\operatorname{Var}[Q]=\operatorname{Var}[R]+\left(1+E\left[X_{e}\right]\right) \cdot \operatorname{Var}[A]+\operatorname{Var}\left[X_{e}\right] \cdot E^{2}[A]
$$

Let us now compute the above mentioned terms. First, the distribution of $X_{e}$ is to be obtained. The distribution of the queue, seen by an admitted arriving packet, is derived from the distribution $P_{q}(i)$ already obtained for local buffers at each timeslot right after a possible transmission. Note that an output is not possible when the queue is empty and no packet can leave behind it $N_{q}$ packets. Hence, we have:

$$
P_{q}\left(X_{e}=k\right)=1\left(0 \leq k<N_{q}\right) \cdot \frac{P_{q}\left(X_{-}=k+1\right) \cdot \Omega_{q, k+1}(1)}{\sum_{i=1}^{N q} P_{q}\left(X_{-}=i\right) \cdot \Omega_{q, i}(1)}
$$

where $X_{-}$is the number of clients in the queue at the end of each timeslot, right before any possible transmission occurs. We have computed in section 3.2 the queue distribution at the end of each timeslot, right after a possible service of the client at the head of the queue. This distribution is related to the one of $X_{-}$by the equations:

$$
P_{q}(X=n)=\left\{\begin{array}{l}
\sum_{i+j=n} P_{q}(i) \cdot \alpha_{q}(j) \text { for } 0 \leq n \leq N_{q}-1 \\
\sum_{i+j \geq N_{q}} P_{q}(i) \cdot \alpha_{q}(j) \text { for } n=N_{q}
\end{array}\right.
$$

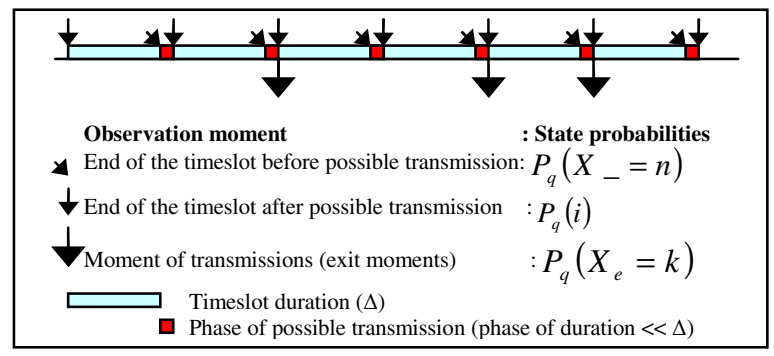

Fig. 3. Observation moments for the studied system

To obtain the expression of the moments of the access time we define first $\overline{\Omega_{q}}$ as:

$$
\overline{\Omega_{q}}=\sum_{i=1}^{N_{q}}\left[\Omega_{q, i}(1) \cdot P\left(X_{-}=i\right) / \sum_{i=1}^{N_{q}} P\left(X_{-}=i\right)\right]
$$

Clearly, $\overline{\Omega_{q}}$ gives the availability of a slot on the average. Therefore, the access time for the packet at the head of each queue follows a geometric distribution:

$$
E[A]=\Delta \cdot \overline{\Omega_{q}} \sum_{i=1}^{\infty} i \cdot\left(1-\overline{\Omega_{q}}\right)^{i-1}=\Delta / \overline{\Omega_{q}}
$$

The previous formula includes additionally the transmission time $\Delta$ needed to deposit this packet on the ring. The variance of $\mathrm{A}$ is equal to:

$$
\operatorname{Var}[A]=\Delta^{2} \cdot\left[1-{\overline{\Omega_{q}}}^{2}{\overline{\Omega_{q}}}^{2}\right]
$$


Finally, we calculate the residual time $R$ to obtain the queueing delay. Optical packets arrivals are independent of the synchronized timeslots on the network ring. Since we consider Poisson arrivals, the packet inter-arrival times have therefore an exponential distribution, on each timeslot, truncated by the duration $\Delta$. The distribution function of $R$ is: $f_{R}(\tau)=\lambda_{q} \cdot e^{-\lambda_{q} \cdot(\Delta-\tau)} /\left(1-e^{-\lambda_{q} \cdot \Delta}\right)$, for $0 \leq \tau \leq \Delta$.

The expectation and the variance of $R$ can be easily obtained:

$$
E[R]=\Delta \cdot\left(1-e^{-\lambda_{q} \cdot \Delta}\right)^{-1}-\left(1 / \lambda_{q}\right), \operatorname{Var}[R]=1 / \lambda_{q}{ }^{2}-\left[\Delta^{2} \cdot e^{-\lambda_{q} \cdot \Delta} /\left(1-e^{-\lambda_{q} \cdot \Delta}\right)^{2}\right]
$$

\section{Extraction and Reemission Mechanism}

The PEM mechanism presents the weakness of dropping some best effort packets which may have to be retransmitted later on the network (TCP, etc.), yielding to an extra load in the network. Therefore, we have proposed the following mechanism:

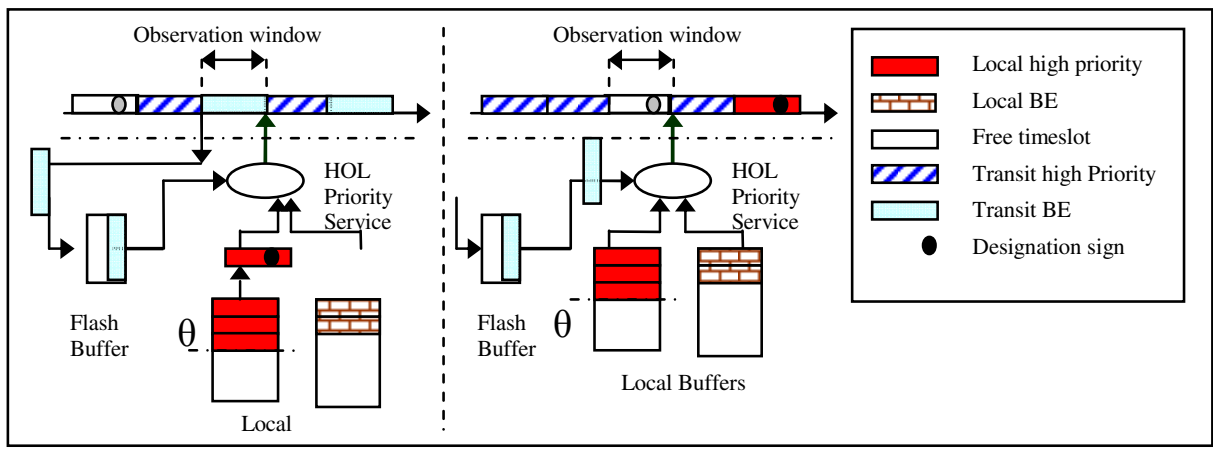

Fig. 4. Example of the ERM mechanism

- A buffer called flash buffer of a very limited capacity is added in order to store few optical Best Effort (BE) packets which can be extracted from the transit line.

- When the current slot at the transit line is occupied by a BE packet, we observe the filling levels of high priority buffers. If one of them reaches a certain threshold $\theta$, the $\mathrm{BE}$ packet at the transit line is preempted and it is stored in the flash buffer. One packet of the high priority overloaded buffer is transmitted instead.

- If the flash buffer is saturated, the extracted BE packet is lost.

- Afterwards, packets waiting in the flash buffer will be retransmitted again, prior to other packets inside the same node, on the first next available timeslots.

\subsection{Modeling of the ERM Mechanism}

In order to model the ERM mechanism, we introduce minor modifications to our model. The flash buffer should be taken into account at three different levels: 
1. The effect of this buffer is reported on the link state at the node entry right after a possible reception

2. The state of this buffer is updated in order to consider possible transmission of one of its packets on the slot

3. When all local buffers are scanned, the state of the flash buffer is updated again in order to consider a possible new arrival (extracted BE packet)

4. Delays and loss expressions for local buffers remain valid. However, some intermediate variables should be added to compute the new state of the system.

The number of packets inside the flash buffer is associated to probabilities denoted $P_{f}$ (n), where $n$ varies from 0 to the flash buffer capacity $N_{f}$. The state of the current slot on the link taking into account the flash buffer is expressed as:

$$
\left\{\begin{array}{l}
P_{l, 1}(0)=P_{l, f}(0) \cdot P_{f}(0) \\
P_{l, 1}(q)=P_{l, f}(q) \quad \text { if } \quad 0<q<C \\
P_{l, 1}(C)=P_{l, f}(C)+P_{l, f}(0) \cdot\left(1-P_{f}(0)\right)
\end{array}\right.
$$

In this case, $P_{l, f}(s)$ denotes the state of the slot seen by the flash buffer right after a possible packet reception at the entry of the considered node. The state of the flash buffer is updated at two levels. The first one, at the node entrance, is as follows:

$$
\left\{\begin{array}{l}
P_{f}^{+}(n)=P_{f}(n) \cdot\left(1-P_{l, f}(0)\right)+P_{f}(n+1) \cdot P_{l, f}(0) \text { for } 0 \leq n<N_{f} \\
P_{f}^{+}\left(N_{f}\right)=P_{f}\left(N_{f}\right) \cdot\left(1-P_{l, f}(0)\right)
\end{array}\right.
$$

According to the thresholds utilization rules, the probability that a Best Effort (BE) packet is extracted is as follows:

$$
P_{E}=P_{l, f}(C) \cdot\left[1-\prod_{q=1}^{C-1} \sum_{0 \leq i+j<\theta_{q}}\left(P_{q}(i) \cdot \alpha_{q}(j)\right)\right]
$$

Thus, the equations for the second update of the flash buffer will be expressed as follows, using the probabilities obtained in eq. 17:

$$
\left\{\begin{array}{l}
P_{f}^{+}(0)=P_{f}(0) \cdot\left(1-P_{E}\right) \\
P_{f}^{+}(n)=P_{f}(n) \cdot\left(1-P_{E}\right)+P_{f}(n-1) \cdot P_{E} \quad \text { for } \quad 0<n<N_{f} \\
P_{f}^{+}\left(N_{f}\right)=P_{f}\left(N_{f}\right)+P_{f}\left(N_{f}-1\right) \cdot P_{E}
\end{array}\right.
$$

The Packet Loss Ratio (PLR) at the flash buffer is equal to the probability of extraction while the buffer is full. Then: $\rho_{f}=P_{f}\left(N_{f}\right) \cdot P_{E}$

When the ERM mechanism is enabled, the total loss ratio of Best Effort packets arriving to a station $v$ can be expressed using the same formula obtained for the PEM mechanism in section 4.1. However, some changes are introduced to the expression of the probability $P_{d \mid C}^{(k)}$ for a Best Effort packet to be dropped (lost) at an intermediate station $k$. It is computed as:

$$
P_{d \mid C}^{(k)}=P_{f}^{(k)}\left(N_{f}^{(k)}\right) \cdot P_{E}^{(k)} / P_{l, f}^{(k)}(C)
$$

Finally, the mean Queueing delay inside the flash buffer: 


$$
E\left[Q_{f}\right]=E\left[n_{f}\right] \times E\left[A_{f}\right]
$$

where $E\left[n_{f}\right]$ is the mean number of packets inside the flash buffer and $E\left[A_{f}\right]$ is the mean access time computed as in section 4.2.

\section{Evaluation and Analysis of the Network Performance}

\subsection{Traffic Assumptions and Node Design Parameters}

Using the ns Network Simulator, we evaluate the performance of a ring optical network with 10 nodes. Given the data provided by our industrial partners, each metro access node can connect about 25 Distant Subscriber Connection Units (DSCU), each of which supports over 2000 subscribers. Therefore, we obtain a total number of 500000 subscribers. As for our model scalability, since we used an iterative approach, we believe that the computation time is a linear function of the number of queues in the network. The same model can be applied to larger networks (e.g. 30 nodes).

The ring consists in one single wavelength of $10 \mathrm{Gbits} / \mathrm{s}$. Unless otherwise mentioned, we consider that the client traffic is uniformly offered to all ring nodes. The load offered to the network (denoted $\rho$ ) is defined as the ratio of the total generated client traffic with regard to the optical link capacity.

Client traffic is classified into four classes of service. To each class corresponds a relative load ratio (with respect to the total load offered to the network) and absolute ratio (percentage of the wavelength capacity required for this traffic).

Table 1. Parameters Related to each Class of Service

\begin{tabular}{|l|l|l|l|l|}
\hline Class & Premium (1) & Silver (2) & Bronze (3) & $\mathrm{BE}(4)$ \\
\hline Rel. ratio & $\mathrm{R} 1=0.25$ & $\mathrm{R} 2=0.25$ & $\mathrm{R} 3=0.25$ & $\mathrm{R} 4=0.25$ \\
\hline Abs. ratio & $\mathrm{A} 1=\mathrm{R} 1 * \rho$ & $\mathrm{A} 2=\mathrm{R} 2 * \rho$ & $\mathrm{A} 3=\mathrm{R} 3 * \rho$ & $\mathrm{A} 4=\mathrm{R} 4 * \rho$ \\
\hline Buffer size & $\mathrm{N} 1=20$ & $\mathrm{~N} 2=20$ & $\mathrm{~N} 3=20$ & $\mathrm{~N} 4=50$ \\
\hline$\theta_{q}$ & $\theta_{1}$ & Disabled & Disabled & Undefined \\
\hline
\end{tabular}

In the simulator of the network, we assume electronic client packets of variable sizes given in [11]. The traffic is constituted as follows: $40 \%$ of packets are of 50 bytes, while the rest consists equally of packets of 500 bytes and 1500 bytes.

Client traffic in the studied model consists of fixed-size optical packets arriving at a Poisson rate. However, in our network simulator, we use the fixed-size packet creation mechanism, which is described in section 2. In this case, optical packets are created from small variable-size packets, with IPP (Interrupted Poisson Process) arrivals. We do not deal in this study with the optical packet creation delay. This topic has been studied in [9]. The timeslot duration $\Delta$ is equal to $10 \mu \mathrm{s}$.

As for performance metrics, we consider the total packet loss ratio (total PLR), the average queueing delay and the squared coefficient of variation for the delay. This last metric is expressed as: $\mathrm{SCV}=\operatorname{Var}[\mathrm{Q}] / \mathrm{E}^{2}[\mathrm{Q}]$. 


\subsection{Model Validation}

We analyze the proposed model with a network load of 0.85 . Each node is supplied with the PEM mechanism $\left(\theta_{1}=5\right)$. Three cases are considered:

Case 1 "Analytical Model": Performance results are obtained using the resolution method proposed in the previous sections.

Case 2 "Model Simulator": We simulate our model with Poisson arrival distributions. This allows validation of the formulas and the resolution of our model.

Case 3 "Network Simulator": This case provides validation of the Poisson arrival assumption. We simulate the original network where electronic client packets arrive according to an IPP distribution. Optical packets are created as described in section 2 .

Simulation results are obtained with $5 \%$ confidence intervals. Figure 5 illustrates average queueing delays and SCV results per class of service. As we can see, average delay and delay SCV are decreasing function of the class priority. Delay SCV is inferior to 1 for all classes of service. Hence we observe that the studied network is a low delay variation system. This is mainly due to the assumed Poisson arrival process and the slotted transmission in the studied model. As for the network simulator, it has been shown in [12] that the fixed-size packet creation mechanism contributes in reducing the traffic burstiness. As for average queueing delays, we observe in Figure5 that these delays vary from $40 \mu$ s for the premium $\operatorname{CoS}$ to about $70 \mu$ s for the best effort traffic. Therefore, with a balanced traffic matrix (which is the case here), the network can assure good performance even for the best effort traffic.
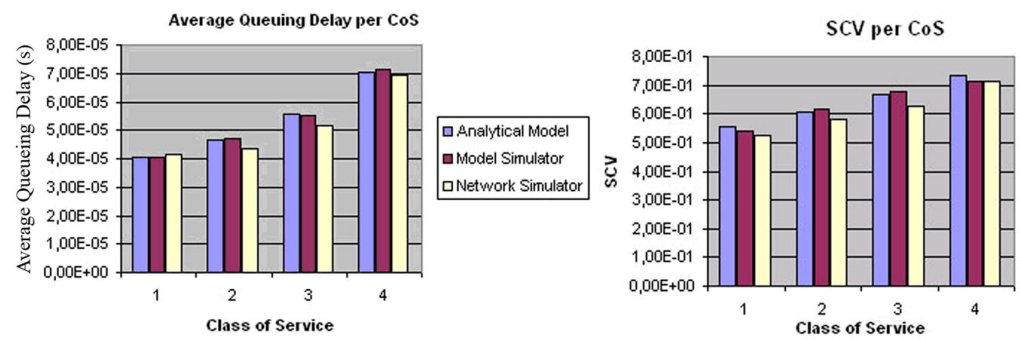

Fig. 5. Model Validation - Average Queueing Delay and SCV

Figure 5 confirms the validity of our model and the Poisson arrival process assumption. The results obtained are quite similar in all studied cases (see Table 2).

Table 2. Concordance of the three studied models (differences in \% with respect to the analytical model)

\begin{tabular}{|l|l|l|l|l|l|l|l|l|}
\hline & \multicolumn{4}{|c|}{ Average Queueing Delays $(\%)$} & \multicolumn{4}{c|}{ SCV (\%) } \\
\hline Class of Service & CoS1 & CoS2 & CoS3 & CoS4 & CoS1 & CoS2 & CoS3 & CoS4 \\
\hline Model Simulator & 0,24 & 1,27 & 1,45 & 1,40 & 2,03 & 1,62 & 1,48 & 2,81 \\
\hline Network Simulator & 3,12 & 6,87 & 7,71 & 1,44 & 5,73 & 5,19 & 6,37 & 2,81 \\
\hline
\end{tabular}




\subsection{Investigation of the PEM and ERM Mechanisms}

In order to investigate the performance of the PEM and ERM mechanisms, we start by a normal function scenario where client traffic is generated according to absolute ratios respectively equal to: $30 \%$ load for premium traffic, $10 \%$ for each of silver and bronze traffic, and $20 \%$ for best effort traffic. The resulting load offered to the network is therefore equal to $70 \%$. Afterwards, we increase the absolute ratio of the premium traffic up to $60 \%$. We compare the following study cases:

- High Load Premium (HLP): In this case, we observe the priority-based Head of Line (HOL) service policy, without intervention of PEM or ERM mechanisms.

- High Load Premium HLP: PEM $\theta_{1}=n$ : The network is still overloaded by additional premium traffic. The PEM mechanism is enabled with $\theta_{l}=n$ packets.

- High Load Premium HLP: ERM $\theta_{1}=3$ : In this case, the ERM mechanism is activated with $\theta_{1}$ set to 3 packets and the capacity of the flash buffer to 2 packets.

The average queueing delay and the PLR per class of service, for each study case, are presented in Figure 6. Because of the Head of Line (HOL) priority scheme, the increase of the load of premium traffic at a given node is translated automatically by simultaneous increase of the average queueing delay at all other buffers in the same node. These delays are reduced by the PEM and ERM mechanisms. PLR results show the counterpart of such delay improvement. For the sake of clarity, loss ratios that are smaller than $10^{-9}$ (obtained by the model) are not shown in this Figure.
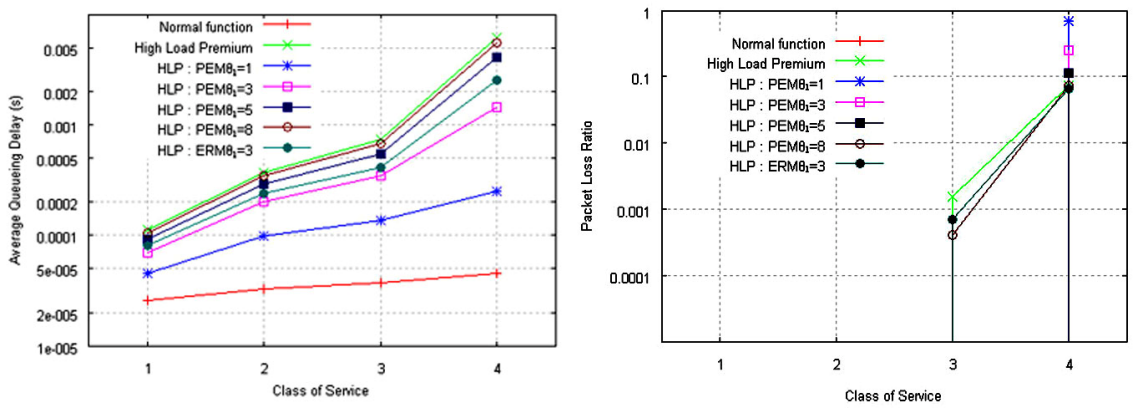

Fig. 6. Average Queueing Delay and PLR per Class of Service (PEM vs ERM)

We observe in the right side of Figure 6 a large loss ratio of BE packets in the case of PEM mechanism with systematic erasing $\left(\theta_{l}=1\right)$. This PLR is reduced when the erasing threshold becomes higher or when we use the ERM mechanism, which preserves the performance of the best effort traffic. Note here that, using the ERM mechanism, Best Effort packets that enter to the flash buffer wait an average delay which is in the order of 4 timeslots (about $40 \mu \mathrm{s}$ ).

\section{Conclusion}

In this work, we provided a performance evaluation of two improved access mechanisms which are applied to a novel Multiservice Optical Packet Switched ring architecture. 
Based on preemptive approaches, these mechanisms implement a sort of dynamic priority. We evaluate the performance of these mechanisms by analytical modeling. Our model captures the impact of the client traffic matrix. Furthermore, it provides expression for the queueing delay and the packet loss measures.

We have used our model in order to evaluate the performance of the PEM and ERM mechanisms. Results obtained have shown that depending on the traffic matrix, the absolute priority scheme is not sufficient to guarantee the performance of the high-priority traffic. On the other hand, we have observed that both PEM and ERM mechanisms contribute in reducing the delay and PLR for the premium traffic. Compared to PEM, the ERM mechanism provides significantly lower Packet Loss Ratios for the Best Effort class (in the local buffer and in transit). Hence, the ERM mechanism provides a good compromise for the priority management in the access nodes of the studied optical ring network.

\section{References}

1. Mathieu, C.: Toward Packet OADM. WDM product group, Alcatel-Lucent (December 2006)

2. Chiaroni, D.: Optical Packet Add/Drop Multiplexers: Opportunities and Perspectives. Alcatel-Lucent R\&I, Alcatel-Lucent presentation (October 2006)

3. Lee, J.Y., Kim, Y.H.: Performance analysis of a hybrid priority control scheme for input and output queueing ATM switches. In: Proceedings INFOCOM 1998 (1998)

4. Bruneel, H., Kim, B.G.: Discrete-time models for communication systems including ATM. Kluwer Academic Publishers, Boston (1993)

5. Lim, Y., Kobza, J.E.: Analysis of a Delay-Dependent Priority Discipline in an Integrated Multiclass Traffic Fast Packet Switch. IEEE Trans. on Com. 38(5) (1990)

6. Maertens, T., Walraevens, J., Bruneel, H.: On priority queues with priority jumps. Performance Evaluation 63, 1235-1252 (2006)

7. Hébuterne, G.: A Gate with Periodic Openings and Bulk Service. In: Teletraffic Science for New Cost-Effective Systems. Elsevier Science Publishers B.V, Amsterdam (1989)

8. Carrier sense multiple access with collision avoidance. American National Standard T1.523-2001, Telecom Glossary (2000), http: / /www.atis.org/glossary/definition.aspx?id=6101

9. Chaitou, M., Hebuterne, G., Castel, H.: On aggregation in almost all optical networks. In: Wireless and Optical Communications Networks, WOCN (2005)

10. Kulkarni, V.G.: Modeling and analysis of stochastic Systems. Chapman and Hall, Boca Raton (1995)

11. IP packet length distribution (2002), http: / /www.caida.org/research/traffic-analysis/AIX/

12. Haciomeroglu, F., Atmaca, T.: Impacts of packet filling in an optical packet switching architecture. In: Advanced Industrial Conference on Telecommunications, AICT 2005 (July 2005) 